Recepción: 19 / 06 / 2019

Aceptación: 22 / 07 / 2019

Publicación: 05 / 08 / 2019
Ciencias de la educación

Artículo de investigación

\title{
El rol de los padres en la inteligencia espiritual de los adolescentes
}

The role of parents in the spiritual intelligence of adolescents

\section{O papel dos pais na inteligência espiritual dos adolescentes}

\author{
Carmen Esther Zambrano-Mendoza ${ }^{\mathrm{I}}$ \\ carmenzm78@hotmail.com \\ Leonor Alexandra Rodríguez-Álava ${ }^{\text {II }}$ \\ alexanroa32@hotmail.com
}

Correspondencia: carmenzm78@hotmail.com

I. Licenciada en Ciencias de la Educación Mención Educación Parvularia, Profesora de Educación Parvularia, Docente de la Universidad Técnica de Manabí, Portoviejo, Manabí, Ecuador.

II. Doctor en Ciencias Pedagógicas, Magister en Desarrollo Educativo, Licenciada en Ciencias de la Educación Especialidad Psicología y Orientación Vocacional, Docente de la Universidad Técnica de Manabí, Portoviejo, Manabí, Ecuador. 


\title{
Resumen
}

Este estudio cuanti-cualitativo, se aborda como una investigación de naturaleza exploratoria, por lo que se trabaja en una pequeña muestra para identificar mediante datos empíricos aspectos fundamentales que proporcionen una visión general, de tipo aproximativo, cuyo objetivo consiste en analizar las percepciones de los padres de familia y adolescentes, en lo referente al rol de los padres respecto al desarrollo de la inteligencia espiritual de los adolescentes; estudio desarrollado en la Unidad Educativa Particular Internacional Puerto de Manta, de la ciudad de Manta, con una muestra de 28 padres y 28 estudiantes de bachillerato. La experiencia investigativa permite observar la potencialidad que existe en los adolescentes para el desarrollo de la Inteligencia Espiritual y la existencia de una actividad consciente de los padres de familia sobre el rol que deben ejercer como familia formadora de sus hijos y de la necesidad de fortalecer el ambiente familiar y el institucional para el logro de personas espiritualmente inteligentes que se respeten a sí mismo y a los demás a través del desarrollo de capacidades que promuevan en el adolescente pensar en la interioridad como una construcción libre, consciente y autónoma, como formas efectivas para afrontar desafíos en su caminar por la vida. Se sugiere continuar investigando el tema y aplicar la experiencia con otros instrumentos $\mathrm{y}$ en diversas poblaciones para fortalecer sus propiedades.

Palabras claves: Inteligencia; inteligencia espiritual; rol de los padres; inteligencias múltiples.

\begin{abstract}
This quantitative qualitative study is approached as an exploratory research, so we work on a small sample to identify, through empirical data, fundamental aspects that provide an approximate overview, whose objective is to analyze the perceptions of parents and adolescents, in relation to the role of parents in the development of adolescent spiritual intelligence; study carried out in the International Private Education Unit Puerto de Manta, in the city of Manta, with a sample of 28 parents and 28 high school students. The investigative experience allows us to observe the potential that exists in adolescents for the development of Spiritual Intelligence and the existence of a conscious activity of the parents about the role that they should play as a formative family of their children and the need to strengthen the family and institutional environment for the achievement of spiritually intelligent people who respect themselves and others through the development of capacities that promote the adolescent to think of interiority as a free, conscious and autonomous construction, as effective ways
\end{abstract}


to face challenges in your walk through life. It is suggested to continue researching the subject and apply the experience with other instruments and in different populations to strengthen their properties.

Key words: Intelligence; spiritual intelligence; role of parents; multiple intelligences.

\section{Resumo}

Este estudo qualitativo quantitativo é abordado como uma pesquisa exploratória, por isso trabalhamos com uma pequena amostra para identificar, através de dados empíricos, aspectos fundamentais que proporcionam uma visão aproximada, cujo objetivo é analisar as percepções de pais e adolescentes, em relação ao papel dos pais no desenvolvimento da inteligência espiritual do adolescente; estudo realizado na Unidade Internacional de Educação Privada Puerto de Manta, na cidade de Manta, com uma amostra de 28 pais e 28 estudantes do ensino médio. A experiência investigativa nos permite observar o potencial que existe nos adolescentes para o desenvolvimento da Inteligência Espiritual e a existência de uma atividade consciente dos pais sobre o papel que devem desempenhar como uma família formadora de seus filhos e a necessidade de fortalecer a ambiente familiar e institucional para a realização de pessoas espiritualmente inteligentes que respeitem a si e aos outros através do desenvolvimento de capacidades que promovam o adolescente a pensar a interioridade como uma construção livre, consciente e autônoma, como formas efetivas de encarar desafios em sua caminhada pela vida. Sugere-se continuar pesquisando o assunto e aplicar a experiência com outros instrumentos e em diferentes populações para fortalecer suas propriedades.

Palavras-chave: Inteligência; inteligência espiritual; papel dos pais; inteligencias multiplas. 


\section{Introducción}

La comprensión de la inteligencia ha sido y sigue siendo tema de interés en las diversas áreas del conocimiento; por mucho tiempo, se ha determinado que las características relacionadas con la inteligencia que pertenecen al ser humano están donde se desarrolla una medición de la capacidad lógico-matemática y verbal, en base a una serie de test estandarizados, al resultado de esta medición se denominó cociente intelectual, valor técnico que representa la racionalidad instrumental y la capacidad de ejercer un control de su entorno.

La literatura consultada, evidencia varias definiciones sobre inteligencia; así: Teijero (2015), la define como la capacidad de asimilar, guardar, elaborar información y utilizarla en resolver problemas; es decir, como la facilidad para aprender, aprehender o comprender, además como la percepción, intelecto e intelectualidad y capacidad para resolver situaciones problemáticas. Por otra parte, (Gardner, 2000) señala que la inteligencia, es una medida singular de competencia que debe desaparecer, debido a que los seres humanos tienen una gama de competencias, denominadas inteligencias múltiples, que existen en diferentes proporciones en distintas personas, estas son: la visual - espacial, lógico matemática, musical, verbal lingüística, corporal - cinestésica, intrapersonal, interpersonal y naturista.

Los trabajos de Howard Gardner no quedaron en su modelo de ocho inteligencias múltiples cuando habla de una novena inteligencia, la de las grandes preguntas, que se refleja en los niños, con una mayor acentuación, debido a que ellos preguntan sobre todo lo que le rodea a través de ¿por qué?; mientras que los adultos meditan sobre la muerte, el amor, el conflicto, el futuro del planeta, es decir, de cuestiones existenciales. De la teoría de afirma: que los seres humanos poseen varias inteligencias relativamente autónomas y que los hace seres humanos, que no existen dos individuos que tengan el mismo perfil de inteligencias, $\mathrm{y}$, el tener una inteligencia no significa que uno se comportará moral o inteligentemente (Gardner, 2000)

En este sentido, afirma Marina (2012), (2015), que esta inteligencia es la encargada de organizar a las demás inteligencias y el propósito es dirigir bien la acción; además que existe un modelo de inteligencia estructurado en dos niveles, la primera denominada, inteligencia ejecutiva, que organiza a todas las demás inteligencias y tiene como objetivo dirigir bien la acción, desde los conocimientos y las emociones, se relaciona con las operaciones mentales que permite plantear objetivos, elaborar proyectos y organizar la acción para realizarlos. La segunda es la inteligencia creadora, que trata de 
resolver un problema de una manera nueva y eficiente. Es la capacidad para descubrir metas, resolver problemas, inventar salidas cuando parece que no las hay, evitar la rutina, el aburrimiento o la desesperanza.

Por otra parte, (Buzan, 2008) define la inteligencia social como la inteligencia que permite crear capacidades para adentrarse en el modo de vida de la gente apreciando en profundidad su comportamiento ante la sociedad. Según Goleman (2010b), la inteligencia social permite crear capacidades para que la gente coopere en el intento de comprender su comportamiento. Según Emmons et al. (2004), es un tipo más de inteligencia, que está compuesta por cuatro componentes: a) capacidad para trascender b) capacidad para experimentar estados de conciencia elevados, c) capacidad para dotar de un sentido de sacralidad a las actividades, acontecimientos; e) capacidad de relaciones cotidianas; así como, la capacidad para utilizar recursos espirituales para solucionar problemas

En concordancia a lo descrito, se puede inferir que el desarrollo de cómo se concibe a las inteligencias puede ser visto desde distintos puntos de vista: uno marcado por los trabajos de Gardner (2000) y su modelo de inteligencias múltiples; otro analizado en los trabajos desarrollados por Goleman (2010a), en su best seller sobre inteligencia emocional, inspirado por el trabajo de Gardner sobre la inteligencia interpersonal e intrapersonal; y otro, que se convierte en el objeto de estudio de este artículo, que es la inteligencia espiritual. Inteligencia relativamente nueva y a la vez antigua. Reportes específicos de la inteligencia espiritual datan desde hace 2.500 años, cuando el Buda la llamó visión cabal o Vipassana. En esta época, esta inteligencia fue vista como la capacidad de visión penetrativa para comprender la realidad profunda de los fenómenos y ver las cosas como son. Esta comprensión fue la base para superar la ignorancia, base del sufrimiento y alcanzar la verdadera felicidad (Martínez-Lage, 1999, p.74)

Torrealba (2014), manifiesta que esta inteligencia enseña a las personas cómo vivir sus vidas de una manera espiritualmente inteligente. Define la inteligencia espiritual como el modo definitivo de conocimiento. La utiliza para imaginar posibilidades, entender el dolor, para responder las cuestiones filosóficas básicas sobre la vida y para encontrar sentido tanto temporal como existencial.

Macías (2015), refiere que la inteligencia espiritual es una capacidad para afrontar y utilizar el sufrimiento, para trascender el dolor, cualidad para ser inspirado por visiones y valores, renunciar a causar un daño innecesario, tendencia a ver conexiones entre diversas cosas, una marcada tendencia 
a preguntas que conducen a respuestas fundamentales, así como capacidad para trabajar en contra de lo convencional.

Varias son las aportaciones relacionadas con la inteligencia espiritual, que permiten comprender su importancia, características y el aporte que ofrece al desarrollo de las personas; uno de los aspectos, que a criterio de las autoras de este artículo es que ella difiere del concepto de religiosidad, con el que se puede confundir; no es lo mimos, aunque se nutren entre sí. Al respecto (Arias y Lemos, 2015) refieren que la inteligencia espiritual es un constructo conformado por tres dimensiones:

a. Conocimiento espiritual: implicaría la actividad cognitiva para la construcción de un concepto trascendente de la existencia y un sistema axiológico correspondiente.

b. Vivencia espiritual: implicaría la experiencia afectiva resultante del conocimiento espiritual.

c. Contingencia: implicaría la coherencia conductual con el sistema axiológico construido mediante el conocimiento espiritual.

La inteligencia espiritual es aquella con la que no sólo se reconocen los valores existentes, sino que creativamente se descubren nuevos valores. Esta tiene que ver con la rectitud, la conciencia, el discernimiento, la veracidad, el significado iluminado del yo y la sabiduría. Para Vásquez (2018), esta inteligencia es la que permite afrontar problemas y poner los actos y cualidades al espíritu. A criterio de (3Q Institute, 2019) esta inteligencia es la base necesaria para el eficaz funcionamiento, tanto de la inteligencia intelectual, como de la inteligencia emocional, ya que el cerebro está diseñado de modo que las inteligencias múltiples funcionen juntas y se complementen.

Como se ha descrito, la inteligencia espiritual, es tema de análisis de estudiosos de esta área del conocimiento; para efectos de este estudio, se la trabaja relacionada con la vida de las personas en la etapa de la adolescencia; aspecto que ha sido investigado por (Johnson y Boyatzis, 2006), quienes argumentan que los adolescentes tienen una comprensión espiritual intuitiva que es andamiada no sólo por el pensamiento reflexivo, sino también por las interacciones familiares, sociales y culturales. Esta espiritualidad innata es obstaculizada en los adolescentes por la falta de lenguaje para expresarla; razón por la cual, resulta oportuno atender la relación entre el desarrollo de la espiritualidad y la calidad de la maduración moral de los jóvenes, desde la cual se adquiere experiencia en cuanto al aprendizaje de ellos, al consolidarse patrones de motivación intrínseca y de estimulación espiritual. 
Para responder a la relación entre el desarrollo de la espiritualidad y la calidad de la maduración moral de los jóvenes.

En cuanto al rol de los padres en el desarrollo de esta inteligencia, abordaremos desde la responsabilidad que tiene la familia en cuanto a la formación integral de sus hijos; así, Echeverría \& Martínez (2015):

“... las buenas prácticas han de tener su inicio alli donde el futuro ciudadano nace y recibe los primeros cuidados, atenciones, mensajes educativos y procesos de interacción y comunicación compartida. Es decir, en el seno de la familia,...” (p.7)

Así lo reconocen muchos autores, y al mismo tiempo enumeran las dificultades que tiene la familia para poder educar a sus hijos, especialmente a los adolescentes, que muchas veces manifiestan conflictos y crisis propias de la edad; y es ahí donde interviene la sociedad a través de las instituciones educativas, donde estas se conviertan en soporte para las familias, ambas instituciones también deben dar respuesta a las necesidades que produce una sociedad y promover un efectivo acercamiento que permita incidir sobre los niños y jóvenes. La formación de los padres forma parte de la educación de los niños y es un método para promover su desarrollo, donde las familias tendrán que asumir que la educación es una labor de todos, como agentes no encargados de aconsejar o proporcionar información; sino, más bien, responsables de transmitir valores, estrategias y técnicas para que los propios miembros resuelvan sus problemas a través de un proceso de desarrollo personal, Cataldo, (1991), Rodríguez (2013)

En cuanto al rol de los padres para el desarrollo de la inteligencia espiritual, parte de considerarla como sabiduría que va más allá del ego o de la mente consciente, que le permita al adolescente afrontar cuestiones sobre el bien y el mal; considerando que ellos imaginan posibilidades no realizables: sueñan, anhelan, se proyectan y se levantan del lodo. Es la inteligencia con la que afronta y resuelven problemas de significados y valores; con la que ponen sus actos y vidas en un contexto amplio y significativo. Los aportes de Medel (2015), estipulan que el trabajo espiritual que tienen los padres es enseñar a que sus hijos como seres humanos se comprendan en una sociedad como condición y garantía de la solidaridad intelectual y moral de la humanidad. Esto encierra la voluntad de trascender lo efímero y lo inmediato; trabajar en la inteligencia espiritual de los adolescentes consiste en 
beneficiarse del potencial que tienen estos y así despertar su curiosidad y avidez por explorar, esto conducirá al joven a ser dueño de su propia vida. Este despertar interior no puede ser transmitido sino por la vía de la experiencia (Mendel, 2015, p.24); es decir que, si una familia que vive en espiritualidad, desarrollará un ambiente propicio para la formación y desarrollo de esta inteligencia, sin que sea impuesta, sino vivida desde el ejemplo de sus padres y familiares. Una sociedad que no potencie los valores profundos que componen la esfera espiritual, generará una sociedad espiritualmente analfabeta, Pérez (2016).

En concordancia a lo analizado, resulta preciso indicar las características de una persona espiritualmente inteligente, para lo cual se ha tomado lo propuesto por Torralba (7-2-2010), quien indica entre otros aspectos: a) Busca profundidad en las relaciones; b) Trata de ver aquello que la une a las otras personas; c) Relativiza las diferencias; d) Goza intensamente de la belleza que se revela en el mundo; e) Comprende que no es el único ser en la tierra; f) Posee capacidad de conexión con el resto; g) Experimenta el deseo de darse tal como es, sin complejo ni sentimiento de culpa.

Siguiendo la misma línea, con el aporte de Torralba (5-10-2010 y 5-03-2010), en cuanto a cómo conseguir el desarrollo de la Inteligencia Espiritual, propone: a) Practicar la soledad ocasional; b) Disfrutar o valorar del silencio físico e interior; c) Intentar alcanzar una comprensión profunda de las cosas; d) Gozar de la faceta espiritual en el arte; e) Ejercer la solidaridad; f) Hablar en profundidad con los demás; g) Leer a los grandes maestros espirituales de la Humanidad; h)Practicar ejercicio físico con regularidad.

Desde el criterio de las autoras de este artículo, el desarrollo de la Inteligencia Espiritual es difícil mas no imposible, exige el respeto a sí mismo y a lo que existe alrededor de cada uno, como base de la misma; exige la meditación que permita transcender a través de la meditación sobre los diversos acontecimientos que nos van sucediendo en la cotidianidad y así hacer frente a las contaminaciones emocionales a las que nos encontramos expuestos en la sociedad actual. Al estar por encima de los demás tipos de inteligencia, tal como los sostiene Covey (2005) y Torralba (2010), es una especie de dinamismo que mueve al ser humano a buscar la plenitud, al perfecto desarrollo de todo nuestro ser, a la profundidad y al sentido de lo que hacemos, padecemos y vivimos. Visto así, la IE se convierte en el antídoto a todas las formas de fanatismo, sectarismo o dogmatismo. 


\section{Materiales y Métodos}

Para la realización de este trabajo de carácter cuali-cuanitativo, de tipo exploratorio y descriptivo; se utilizó como métodos y técnicas que coadyuvaron a la escritura de este artículo la sistematización y otros métodos teóricos tales como el inductivo-deductivo, el análisis y la síntesis de la literatura referida al tema, todos los cuales contribuyeron a delinear las bases para el desarrollo de la Inteligencia Espiritual y el rol que cumplen los padres de familia. Se realizó además encuestas semiestructurada a 28 estudiantes y entrevista a 28 padres de familia de la Unidad Educativa Particular Internacional Puerto de Manta donde se desarrolló el estudio, material que propició la validación de los resultados procesados estadísticamente y así establecer las respectivas conclusiones.

\section{Resultados y discusión}

Figura 1. Aplicación de la Inteligencia Espiritual

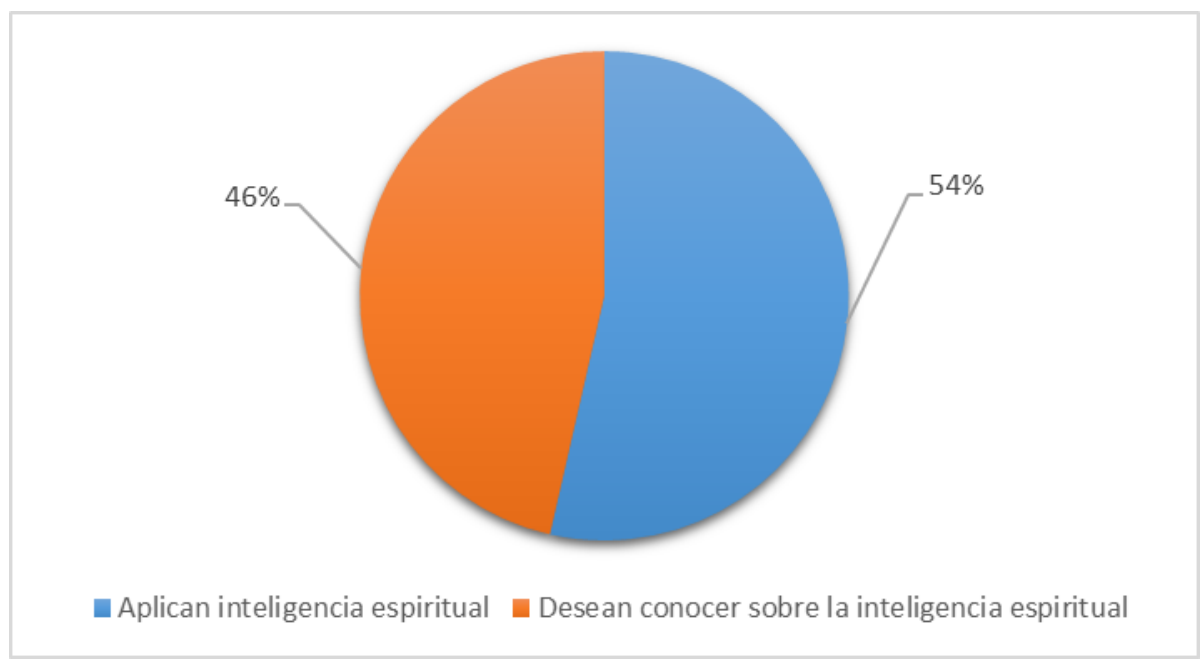

Fuente: Adolescentes de la Unidad Educativa Particular Internacional Puerto de Manta Elaborado por: Investigadoras 
Al consultar a los adolescentes sobre la aplicación de la inteligencia espiritual, se pudo constatar que el $54 \%$ manifiestan que la aplican en su cotidianidad; lo que evidencia que es un tema de interés relacionado con el saber y el ser de los estudiantes y que a criterio de los autores es fácil que los padres apliquen con ellos. Por otra parte, el $46 \%$ de los encuestados desean conocer acerca del tema para poder ganar mayor aplicabilidad. Se denota que es posible obtener avances sobre el desarrollo de la Inteligencia Espiritual, el deseo de conocer en ellos facilita el proceso, da apertura a que padres y maestros puedan intervenir con mayores posibilidades de éxitos.

Lo anterior se refuerza con lo expresado por Zohar y Marshall (2001) quienes afirman que usamos la Inteligencia Espiritual para ser creativos, se recurre a ella cuando se requiere ser flexible, visionario, espontáneo, da sentido a la vida, se la utiliza para lidiar con los problemas existenciales, nos hace conscientes de ellos para poder resolverlos o al menos encontrar paz pese a ellos. En la teoría del caos, el «límite» es la frontera entre caos y orden, entre saber tranquilamente lo que somos y estar absolutamente perdidos. Es el sitio en que podemos ser más creativos.

Figura 2. Indicadores del desarrollo de la inteligencia espiritual en los adolescentes

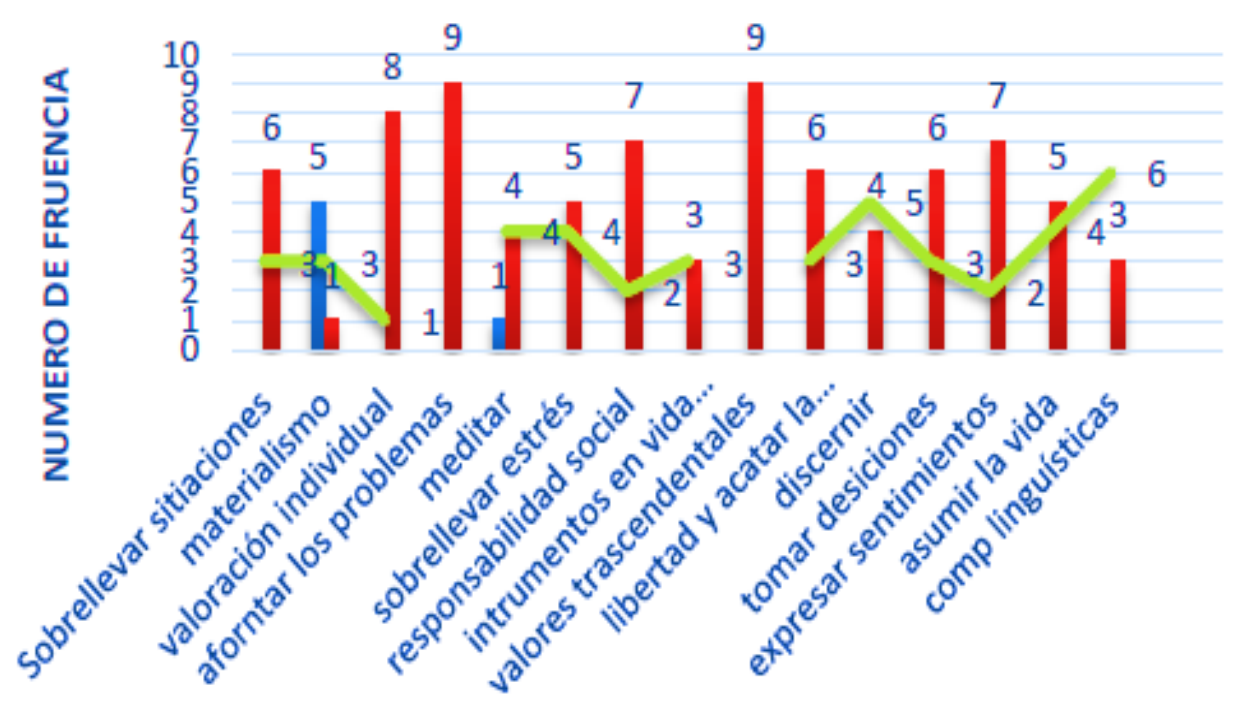

\section{NUNCA $\square$ SIEMPRE $\cong$ V VECES}

Fuente: Adolescentes de la Unidad Educativa Particular Internacional Puerto de Manta Elaborado por: Investigadoras 
Los resultados de la figura dos confirman lo analizado en el gráfico anterior; los estudiantes manifiestan que son capaces de afrontar los problemas y de vivir valores trascendentales con mayor frecuencia de siempre (9), seguida de la valoración individual, expresar los sentimientos y la responsabilidad social (8); mientras que la frecuencia de a veces resalta aspectos como sobrellevar el estrés (5) el discernimiento (4) y las competencias lingüísticas (6); y nunca resalta el materialismo (5) y la meditación. Estos resultados demuestran que los adolescentes encuestados viven de acuerdo con los intereses propios de la edad, de acuerdo con lo afirmado por la Psicología Evolutiva, creen en ellos, en sus fortalezas, por eso afrontan con facilidad los problemas y no se dejan absorber por el estrés y la capacidad de expresar los sentimientos; aspectos que a criterio de los autores se convierte en terreno fértil para potenciar la inteligencia espiritual. Se corrobora que existe facultad humana para aplicar la inteligencia espiritual lo que implica que es un tema usual, renovador y que favorece no solo a los adolescentes en su aprendizaje, sino que favorece el proceso de enseñanza - aprendizaje, ya que esta inteligencia, constituye un área trasversal en la que se integra y se fortalece la vida del estudiante en todas sus dimensiones.

En este aspecto, se concuerda con Teodosio y Rodríguez (2013) en su estudio sobre la Inteligencia espiritual, donde afirma que no hemos sido educados para desarrollar la IE, resalta el interés que tiene su cultivo en la educación de los más jóvenes. Y aquí se presenta una brecha, a través de la cual, se podrían iniciar investigaciones en el campo educativo. Se cree que los estudiantes de Primaria y Educación Media, serían los más indicados para hacerle conocer estas ideas ya que ellos, de por si, en esa edad de adolescencia se hacen muchas preguntas; algunas a veces, desconciertan hasta a sus propios padres, como la de preguntarse: ¿por qué tengo a estos padres y no a otros?; además considera que con la IE se superan barreras y obstáculos que dificultan la comunicación en entornos íntimos, laborales y sociales; se hace necesario pensar en la posibilidad de poner en práctica este nuevo paradigma.

Aspectos que se complementan con lo afirmado por Torralba (5-2-2010), en su artículo "A cultivar la inteligencia espiritual", indica que esta inteligencia sirve para desarrollar la creatividad, obtener una conciencia crítica y autocrítica, mejorar las relaciones interpersonales, afianzarse en la autodeterminación, obtener sentido de los límites, conocimiento profundo de uno mismo, mantener un equilibrio interior, hacer de nuestras vidas, "Un proyecto de Vida", tener la capacidad de sacrificarse en aras de obtener los ideales a los que ha abrazado, obtener una vivencia plena del aquí 
y el ahora, gozarse de la sencillez y vivir plenamente el presente, lo que podría ser un buen antídoto para combatir el Stress.

Figura 3. Actitudes que evidencian inteligencia espiritual

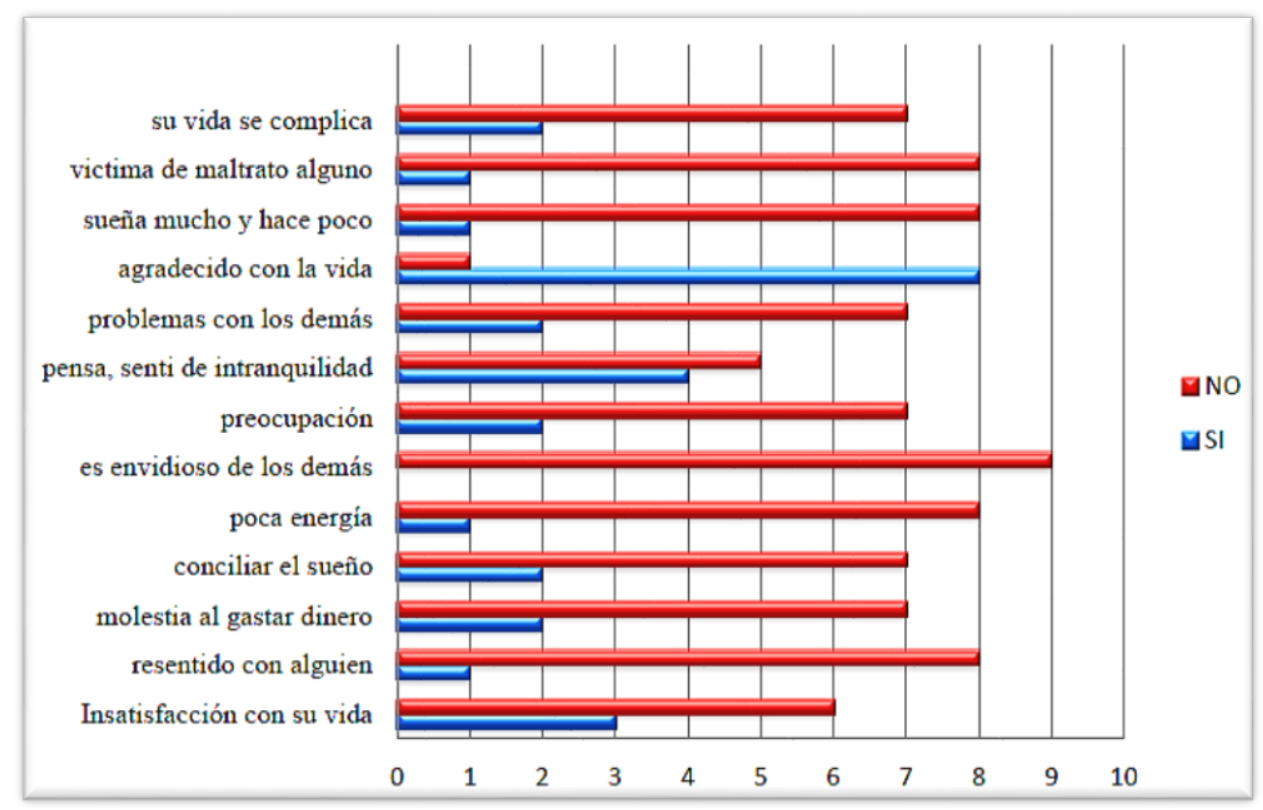

Fuente: Adolescentes de la Unidad Educativa Particular Internacional Puerto de Manta Elaborado por: Investigadoras

De acuerdo con los aspectos más importantes que se desarrollan con la inteligencia espiritual en los adolescentes, se constató a través de la encuesta y que se muestran en la figura 3, actitudes de optimismo para asumir la vida, se consideran personas responsables de su propia formación, de su vida, lo que indica que son personas que pueden ir cultivando su interés por desarrollar la Inteligencia Espiritual y que con ellos es posible educar seres con habilidades que promuevan un mejor nivel de vida y sentido pleno.

Para Robert Cloninger, Przybeck y Svrakic (1994), la espiritualidad es una dimensión de la personalidad que abarca la capacidad de trascendencia del ser humano, el sentido de lo sagrado o los comportamientos virtuosos que son exclusivamente humanos, como el perdón, la gratitud, la humildad o la compasión.

Para corroborar los resultados obtenidos con los estudiantes, se les consultó a los padres de familia sobre: 


\section{Importancia y beneficios que se obtiene con la inteligencia espiritual.}

- La inteligencia espiritual, ayuda a reconocer al ser humano como poseedor de un mundo espiritual, a encontrar sentido de vida y resolver sus inquietudes y a su margen trascendental da respuestas desde la parte ética.

- Se despierta el autorreflexión en los adolescentes, se redescubre su dimensión trascendental, y se despiertan habilidades lingüísticas a partir de las inteligencias múltiples, lo que se complementa con la religiosidad con la que conviven.

- La inteligencia espiritual es una facultad del individuo en la interacción consigo mismo y con el Ser Supremo, la misma sirve para reforzar la parte moral, espiritualidad, vivenciándola a través de las manifestaciones diarias.

- Es un tema que implica fortalecer el derecho a ser persona en su desarrollo con su mundo individual, ecológico, familiar y su entorno social.

\section{Entre las estrategias que los padres determinaron de acuerdo con su rol son los siguientes:}

- Los adolescentes en su casa deben tener una línea de control total sobre qué y cómo son las reglas de convivencia en el hogar.

- Se debe promover para que los papás ocupen un rol activo en la educación de sus hijos. Donde las clases estén diseñadas para que los adolescentes se sientan como en casa.

- Los adolescentes en casa y también en su unidad educativa debe ser capaces de tomar decisiones, así como de asumir ciertos niveles de responsabilidad.

- Se debe implementar tecnología en casa y en la unidad educativa para que los adolescentes consigan una mayor autonomía, posibilitando recursos de estudio, herramientas e incluso comunidades educativas. 


\section{Conclusiones}

Después del análisis de los aspectos teóricos y de los resultados obtenidos en las encuestas a estudiantes y entrevistas a padres de familia, sobre la inteligencia espiritual en los adolescentes y el rol de los padres, se llega a las conclusiones siguientes:

- La Inteligencia Espiritual, a pesar de ser poco reconocida e incluso a veces relacionada con la religiosidad en las personas, es un tema de mucho interés analizada por varios autores y que permite a las personas el desarrollo de capacidades particulares para hacer frente y con sentido de conciencia las adversidades de la vida y que debe ser fortalecida desde la práctica familiar, de manera especial en niños y adolescentes para sentar las bases en la vida adulta.

- En los adolescentes de la Unidad Educativa Particular Internacional Puerto de Manta, existe facultad humana para desarrollar la inteligencia espiritual en los adolescentes, evidenciadas en actitudes que facilitan la misma, a través del proceso de enseñanza - aprendizaje, ya que se cuenta con la conciencia sobre ella tanto en estudiantes como en los padres de familia, quienes están conscientes en la necesidad de ella y la importancia de las normas en la casa y en la escuela para su fortalecimiento.

- El rol de los padres es fundamental en el desarrollo de la inteligencia espiritual de los adolescentes, estos deben asumir la responsabilidad de la formación integral de los hijos, promoviendo un ambiente de respeto y vivencia espiritual, lo que permitirá que se conviertan en personas con proyectos de vida, capacidad de superar los obstáculos y de búsqueda permanente de vivir en paz consigo mismo y los demás. 


\section{Referencias Bibliográficas}

Arias, R. y Lemos, V. (2015). Una aproximación teórica y empírica al constructo de inteligencia espiritual. Enfoques 27, 79-102.

Buzan, T. (2008). El poder de la Inteligencia Espiritual. 10 formas de despertar tu genio espiritual. Madrid: URANO, S.A.

Cataldo, C.Z. (1991): Aprendiendo a ser padres: conceptos y contenidos para el diseño de programas de formación de padres. Madrid: Visor

Celina H. y Campo A., 2005, “Aproximación al uso del coeficiente alfa de Cronbach", Revista colombiana de psiquiatría, vol. XXXIV, número 004, Asociación Colombiana de Psiquiatría, Bogotá, Colombia, pp. 572 - 580, disponible en: http://redalyc.uaemex.mx/pdf/806/80634409.pdf

Cloninger, C.R., Przybeck, T.R., Svrakic, D.M. (1994). The Temperament and Character Inventory (T.C.I.): A guide to its development and us. Washington: Washington University

Echeverría, B., \& Martínez, P. (2015). Orientación en acción. España. Revista electrónica interuniversitaria de formación del profesorado. $51(18,2), 1-14$.

Emmons, R., Cheung, C. y Tehrani, K. (2004). Assessing spirituality through personal goals: Implications for research in religion and subjective well-being. Social Indicators Research, $45,391-422$.

Gardner, H. (2000). The disciplined mind. New York: Penguin Putnam. Leadership \& Organization Development Journal, Vol. $23 \mathrm{n}^{\circ} 2$.

Goleman, D. (2010a). La Inteligencia Emocional. México D.F.: Zeta

Goleman, D. (2010b). Emotional intelligence: issues in paradigm building. Primera edición. San Francisco: Jossey-Bass. ISBN: 978-0787956905.

Hernández Sampieri R.,y otros, 2003, "Metodología de la Investigación”, Editorial Mc. Graw Hill, México D.F. 
Johnson, C. N., \& Boyatzis, C. J. (2006). Cognitive-cultural foundations of spiritual development. En E. C. Roelkepartain, P. Ebstyne King, L. Wagener, \& P. L. Benson (Eds.), The handbook of spiritual development in childhood and adolescence. California: Sage Publications.

Macías, A. (2015). La Inteligencia Espiritual: el despertar hacia la Felicidad Interior. Jalisco: Centro de Capacitación y Calidad Jalisco.

Marina, J. A. (2012). La Inteligencia Ejecutiva. Barcelona: Ariel.

Marina, J. A. (2015). La Inteligencia Ejecutiva. Un enfoque Computacional-Ejecutivo. Universidad Central de Venezuela. Biblioteca up. Ariel.

Martínez-Lage, M. (1999). Dalai Lama. El arte d vivir en el nuevo milenio. Dharamsala: grijalbo mondadori.

Medel Martínez , B. (2015). Propuesta inteligencia alumnado para estimular la espiritual en el de primer ciclo de Educación Primaria. Universidad Internacional de La Rioja . España: UNIR.

Pérez, M. (2016). Inteligencia espiritual. Conceptualización y cartografía psicológica. Revista. PSICOLOGÍA, CIENCIA Y PROFESIÓN: MIRANDO AL FUTURO. 1(2), 294. http://dx.doi.org/10.17060/ijodaep

3Q Institute. (2019). Spiritual intelligence: Spiritual intelligence traing. Recuperado de https://sqi.co

Rodríguez, A. (2013). La relación escuela - familia y las competencias del docente. Revista Sinapsis, Vol. 3, $\mathrm{N}^{\mathrm{o}} 2,1-14$.

Teijero, S. (2015). Es posible multiplicar el poder de la mente. Inteligencia espiritual. Parte X. El poder del amor para fortalecer el espíritu. Décimo artículo de la serie Inteligencia Espiritual: la suprema de las inteligencias.

Teodosio, E y Rodríguez, S (2013). Inteligencia espiritual Teodosio E. Rodríguez S. Sapiens. Revista Universitaria de Investigación, 11-21

Torralba F. (5-10-2010) ¡A cultivar la inteligencia espiritual! [Documento en línea] http://www.venyve.com/vvidayestilo/2010/10/5/a-cultivar-lainteligencia-espiritual.aspx. 
Torralba F. (7-2-2010). Espiritualidad no es huida, es fluidez (Entrevista) [Documento en línea] http://www.avesgams.org/foro/index.php?topic=214.0.

Torrealba, F. (2014). Inteligencia Espiritual. Barcelona: Plataforma Editorial

Vásquez Barragán, M. (2018). La inteligencia espiritual y sus aportes a la Educación Religiosa Escolar en niños de preescolar. Universidad de La Salle, Colombia. Colombia: FSC.

Zohar, D y Marshall, I 2001. Inteligencia espiritual. Plaza \& Janes Editores, S. A. Travessera de Gracia, 47-49. 08021 Barcelona 